\title{
Natural course of congenital hypothyroidism by dual oxidase 2 mutations from the neonatal period through puberty
}

\author{
Yoshihiro Maruo', Keisuke Nagasaki, Katsuyuki Matsui, Yu Mimura, Asami Mori, \\ Maki Fukami ${ }^{2}$ and Yoshihiro Takeuchi \\ Department of Pediatrics, Shiga University of Medical Science, Tsukinowa, Seta, Otsu, Shiga 520-2192, Japan, \\ ${ }^{1}$ Department of Pediatrics, Niigata University, Niigata, Japan and ${ }^{2}$ Department of Molecular Endocrinology, \\ National Research Institute for Child Health and Development, Tokyo, Japan
}

\author{
Correspondence \\ should be addressed \\ to $\mathrm{Y}$ Maruo \\ Email \\ maruo@belle.shiga-med.ac.jp
}

\begin{abstract}
Aim: We previously reported that biallelic mutations in dual oxidase 2 (DUOX2) cause transient hypothyroidism. Since then, many cases with DUOX2 mutations have been reported. However, the clinical features and prognosis of individuals with DUOX2 defects have not been clarified.

Objective: We investigated the prognosis of patients with congenital hypothyroidism (CH) due to DUOX2 mutations. Patients: Twenty-five patients were identified by a neonatal screening program and included seven familial cases. Their serum TSH values ranged from 18.9 to $734.6 \mathrm{mU} / \mathrm{l}$. Twenty-two of the patients had low serum free thyroxine $\left(\mathrm{fT}_{4}\right)$ levels (0.17-1.1 ng/dl). Twenty-four of the patients were treated with L-thyroxine.

Methods: We analyzed the $D U O X 2$, thyroid peroxidase, $\mathrm{Na}^{+} / \mathrm{I}^{-}$symporter, and dual oxidase maturation factor 2 genes of these 25 patients by PCR-amplified direct sequencing. An additional 11 genes were analyzed in 11 of the 25 patients using next-generation sequencing.

Results: All patients had biallelic DUOX2 mutations, and seven novel alleles were detected. Fourteen of the patients were able to discontinue replacement therapy, and seven were receiving reduced L-thyroxine doses. Normalization of thyroglobulin lagged several years behind the completion of treatment. Two patients showed permanent hypothyroidism. Except for one case of a learning disability, growth and psychomotor development were normal.

Conclusion: The prognosis of Japanese patients with DUOX2 defects was usually transient $\mathrm{CH}$. Delayed improvement of thyroglobulin indicates that these patients have subclinical hypothyroidism. Hypothyroidism did not recur in patients during the study period (up to 18 years old).

European Journal of

Endocrinology

(2016) 174, 453-463
\end{abstract}

\section{Introduction}

Congenital hypothyroidism $(\mathrm{CH})$ is one of the most common endocrine diseases in infants. The incidence of $\mathrm{CH}$ reported from worldwide neonatal screening programs is between $1 / 2000$ and $1 / 4000(1,2)$, and its etiology is heterogeneous. The most common cause of $\mathrm{CH}$ is thyroid dysgenesis (ectopy, athyreosis and orthotopic hypoplasia), which accounts for $75-80 \%$ of cases (3). Defects in thyroxine synthesis account for 15-20\% of cases (3). However, the etiology of many cases is unknown because the underlying cause is not clear.

Recently, the mechanisms of thyroid hormone production and the associated genes have been clarified. The genes associated with thyroid hormone production include thyroid peroxidase (TPO), thyroglobulin (TG), 
sodium iodide symporter (NIS), pendrin (PDS), dual oxidase 2 (DUOX2), dual oxidase maturation factor 2 (DUOXA2), dual oxidase 1 (DUOX1), dual oxidase 1 maturation factor (DUOXA1), and iodotyrosine deiodinase (IYD) $(4,5,6,7,8,9,10,11,12)$, and mutations have been detected in numerous undiagnosed cases. Recent reports have shown that a high proportion of $\mathrm{CH}$ cases are caused by mutations in hormone-producing enzymes, and DUOX2 mutations are one of the most frequent causes $(13,14,15,16)$.

DUOX2, which is also known as thyroid oxidase 2 (THOX2) $(10,17)$, spans 6376 nucleotides on chromosome 15 and includes 33 exons. The encoded DUOX2 protein is a 1,548-amino acid polypeptide that includes a 26 -amino acid signal peptide. DUOX2 is localized to the apical membrane of thyrocytes, and it is involved in the $\mathrm{Ca}^{2+} / \mathrm{NADPH}$-dependent generation of $\mathrm{H}_{2} \mathrm{O}_{2}$. To synthesize thyroid hormone, TPO requires $\mathrm{H}_{2} \mathrm{O}_{2}$ to catalyze both the iodination of tyrosine residues and the coupling of iodotyrosine residues of TG (18).

In 2002, Moreno et al. (8) demonstrated that DUOX2 defects cause $\mathrm{CH}$. They found that patients with homozygous DUOX2 mutations showed permanent $\mathrm{CH}$, and patients with heterozygous mutations showed transient $\mathrm{CH}$. Since then, additional $\mathrm{CH}$ cases with underlying DUOX2 mutations have been reported $(19,20,21)$. In 2008, we reported eight cases of transient $\mathrm{CH}$ with biallelic DUOX2 mutations (13). Ohye et al. reported that a homozygous missense mutation (p.R1110Q) causes acquired goiterous hypothyroidism (22). Narumi et al. showed that DUOX2 mutation is the most prevalent cause of $\mathrm{CH}$ due to dyshormonogenesis (14). However, many questions remain regarding DUOX2 mutations, the genetic mechanisms, and clinical course of $\mathrm{CH}$. In this study, we examined 25 Japanese patients with $\mathrm{CH}$ caused by biallelic DUOX2 mutations including follow-ups until up to 18 years of age.

\section{Patients and methods}

\section{Patients}

Twenty-five patients with biallelic DUOX2 mutations were included in the study. Twenty-one patients were female, and four were male. Their present ages range from 3 to 18 years. There were seven familial cases (family 1 , cases $1-4$; family 2, cases 5 and 6; family 3 , cases 8 and 9; family 4, cases 10 and 11; family 5, cases 13 and 14; family 6, cases 16 and 17; and family 7, cases 24 and 25), which included two sets of twins (family 4 (monozygotic twins) and family
6 (dizygotic twins)). All familial cases were siblings. All patients were identified based on elevated thyroid stimulating hormone (TSH) levels in a neonatal screening program, and consequently visited our hospital. All the infants had a gestational age $>35$ weeks (range, 35 weeks 4 days to 41 weeks 3 days; mean, 38 weeks 4 days \pm 11.7 days). Their birth weight and length ranged from 2406 to $3460 \mathrm{~g}$ (mean, $2870 \pm 290 \mathrm{~g}$ ) and $44.0-50.0 \mathrm{~cm}$ (mean, $47.4 \pm 1.4 \mathrm{~cm}$ ), respectively. The observed birth weights and lengths were appropriate for the gestational ages of the patients (Table 1). The TSH concentration at neonatal screening ranged from 10.0 to $242.8 \mu \mathrm{U} / \mathrm{ml}$ (median, $32.75 \mu \mathrm{U} / \mathrm{ml}$ ). Serum concentrations of TSH, free tri-iodothyronine $\left(\mathrm{fT}_{3}\right)$ and free $\mathrm{T}_{4}\left(\mathrm{fT}_{4}\right)$ were $25.7-$ $771.0 \mu \mathrm{U} / \mathrm{ml}$ (median, $94.0 \mu \mathrm{U} / \mathrm{ml}$ ), 1.89-5.52 $\mathrm{pg} / \mathrm{ml}$ (median, $3.72 \mathrm{pg} / \mathrm{ml}$ ) and $0.18-1.50 \mathrm{ng} / \mathrm{dl}$ (median, $0.55 \mathrm{ng} / \mathrm{dl}$ ), respectively (Table 1 ). TG concentrations were very high, and in most cases, > $800 \mathrm{ng} / \mathrm{ml}$, except in two patients (family 7, patients 24 and 25, with 150 and $91.8 \mathrm{ng} / \mathrm{ml}$, respectively). Using a checklist for clinical diagnosis based on the mass-screening guidelines for $\mathrm{CH}$ of the Japan Society of Pediatric Endocrinology and Japan Society of Mass Screening, 12 of 23 patients scored above two points for symptoms of $\mathrm{CH}$ (e.g., prolonged jaundice, constipation, umbilical herniation, failure to thrive, dry skin, poor activity, macroglossia, hoarseness, cold extremities, edema, opened posterior fontanellae and goiter; more than two points indicates severe $\mathrm{CH}$ ). Thyroid ultrasonography showed enlargement of the thyroid gland in 11 of the 21 patients. The other ten patients had an orthotopic thyroid gland without enlargement. Six of the 25 patients showed an absence of distal femoral epiphysis (Table 1).

Except for one patient with transient hyperthyrotropinemia in family 1 , all patients were treated with L-thyroxine. The first doses of L-thyroxine were determined by the severity of hypothyroidism (check list, elevation of $\mathrm{TSH}$ level and reduction of $\mathrm{fT}_{4}$ level) at first visit to our outpatient department. The usual dose is $5-10 \mu \mathrm{g} / \mathrm{kg}$ per day. However, case 6 did not show apparent reduction in serum $\mathrm{fT}_{3}$ and $\mathrm{fT}_{4}$ levels; a lower dose was used.

The reevaluation and classification of patients as having transient or permanent $\mathrm{CH}$ were based on normalization of TSH levels despite withdrawal of L-thyroxine. After withdrawal of therapy, serum $\mathrm{TSH}, \mathrm{fT}_{3}, \mathrm{fT}_{4}$ and TG levels of all patients were followed once or twice a year until now (Table 2).

This study was approved by the ethics committee of Shiga University of Medical Science. 


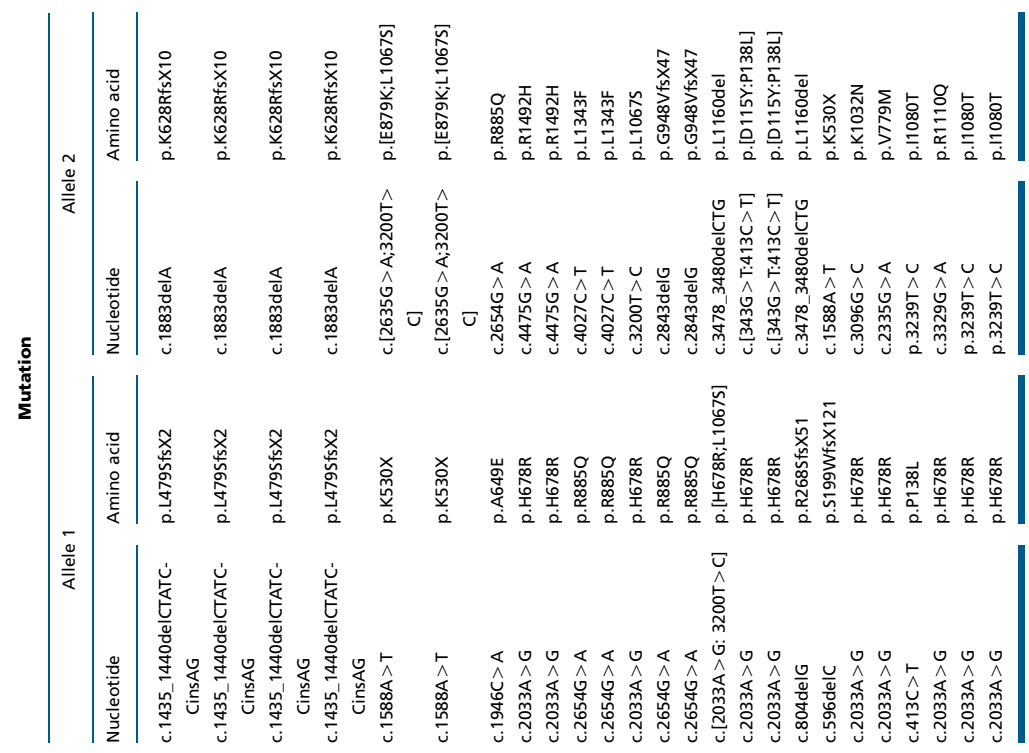

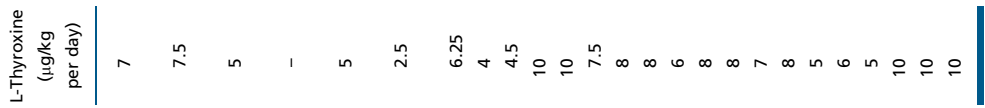

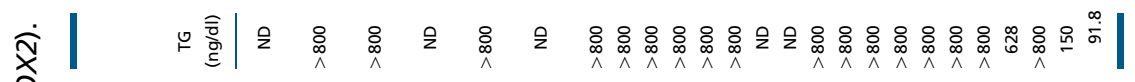

可| E

导

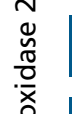

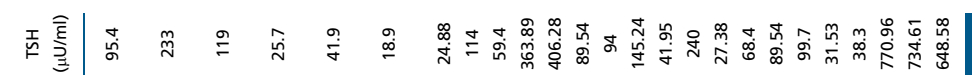

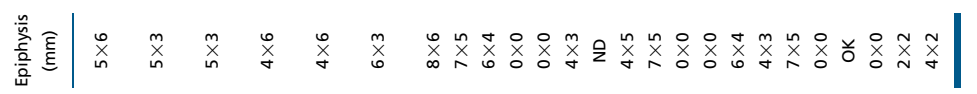

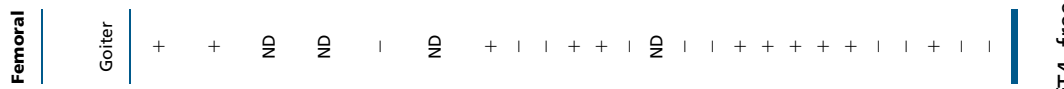

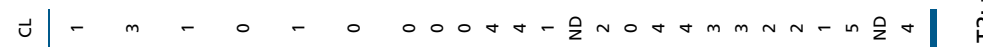

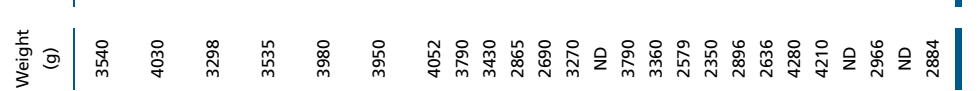

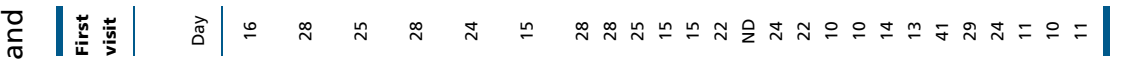

党

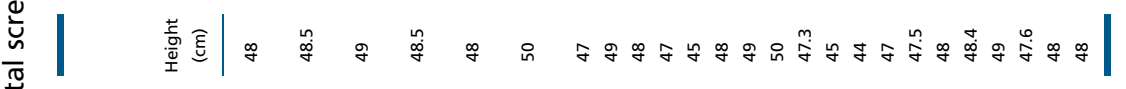

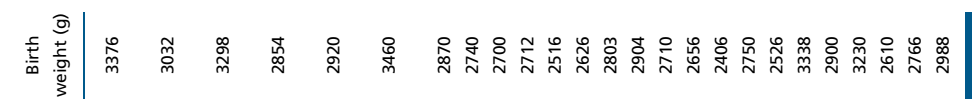

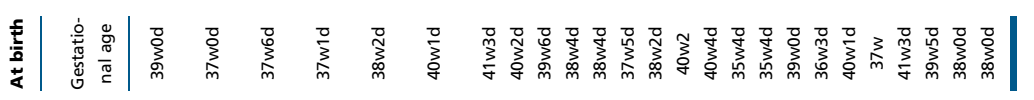

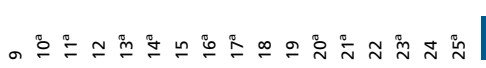




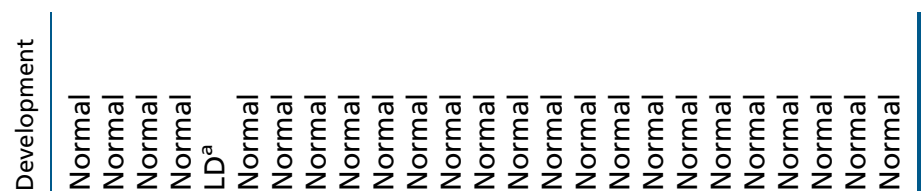

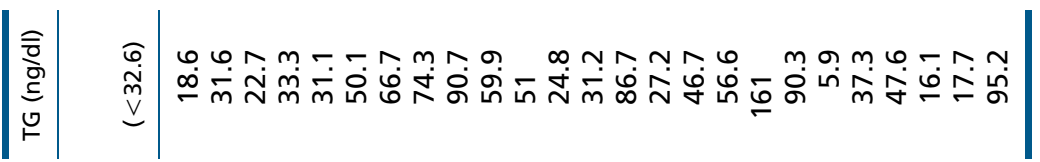

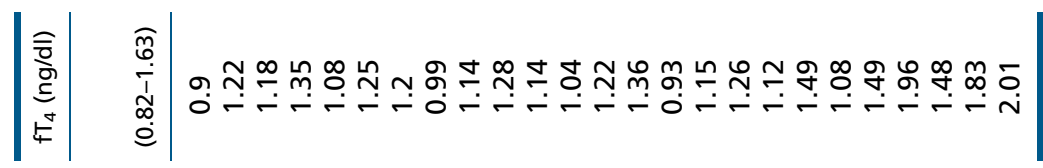

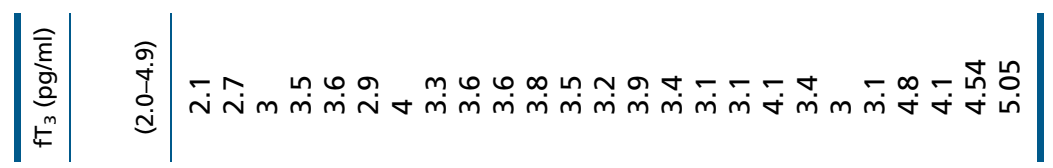

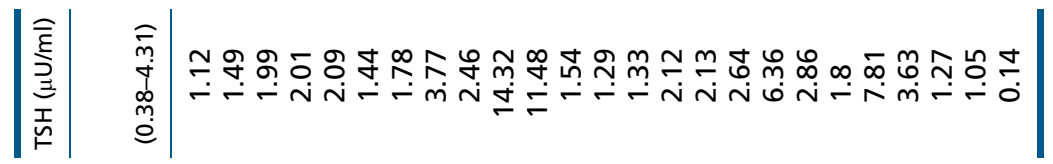

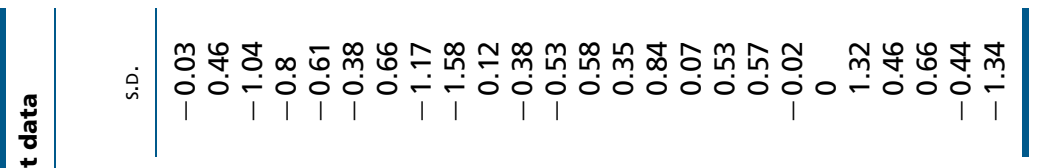

ह

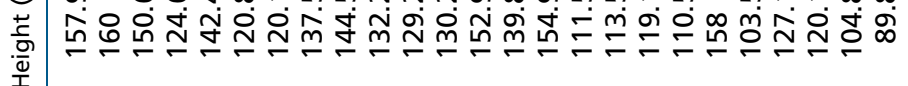

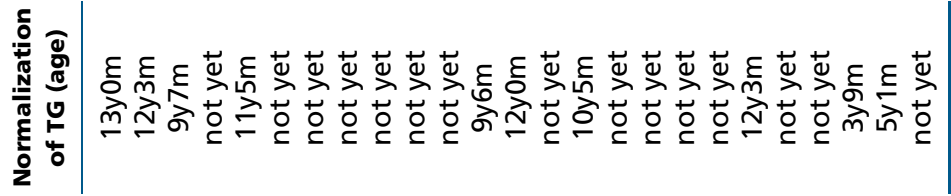

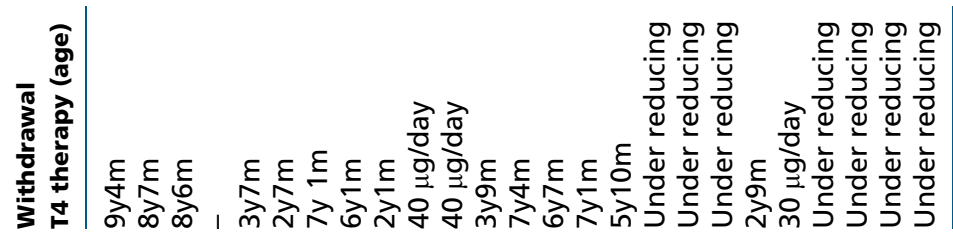

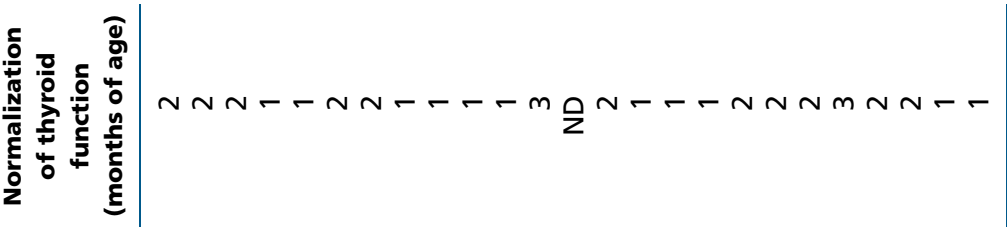

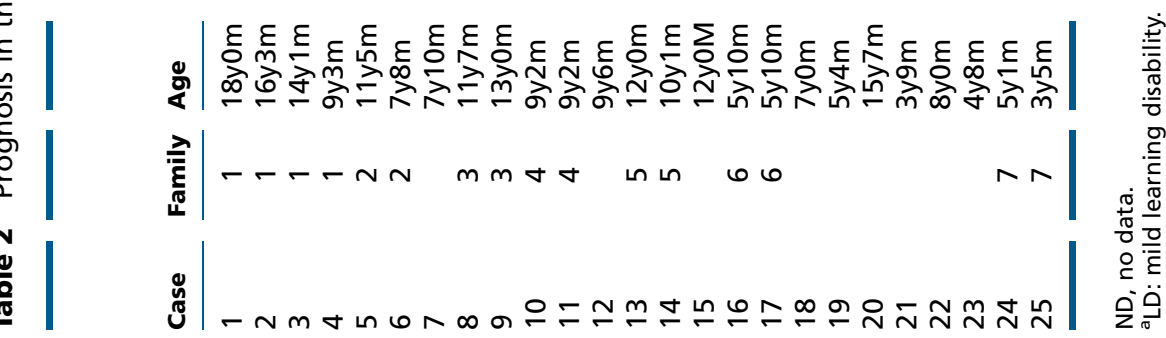




\section{Laboratory testing and sequence analysis}

At neonatal screening, TSH was measured by the Cretin TSH ELISA II assay (Eiken Chemical, Tokyo, Japan). During hospital visits, TSH was measured using a fluorescent enzyme immunoassay method (TOSHO, Tokyo, Japan). $\mathrm{fT}_{4}$ and $\mathrm{fT}_{3}$ levels were measured with a fluorescent enzyme immunoassay (TOSHO). TG was measured with an immunoradiometric assay (Eiken Chemical).

Genomic DNA was extracted from blood leukocytes using standard techniques after obtaining informed consent from patients and their families to participate in this study. Amplification of exons and exon-intron boundaries by PCR from genomic DNA was performed using the ten pairs of oligonucleotide primers shown in Supplementary Table 1 , see section on supplementary data given at the end of this article (13). The genomic DNA sequences and CDNA sequences were based on those in the human genome database (NCBI accession numbers CCDS10117.1 and AF267981). The 33 exons of DUOX2 were amplified as ten PCR products. PCR products were purified using the Nucleospin Gel \& PCR Clean-up Kit (TaKaRa, Kyoto, Japan), and the sequences of the amplified DNA fragments were determined directly using the BigDye Terminator v1.1 Cycle Sequencing Kit (Applied Biosystems) and an ABI PRISM 3130xI Genetic Analyzer (Applied Biosystems). The sequencing primers are shown in Supplementary Table 1.

The amplified DUOX2 fragments were subcloned into the pCR 2.1 vector using the TA Cloning Kit (Invitrogen) to verify the mutant sequence in patients with deletion or insertion mutations, and to determine the cis or trans arrangement of the two mutations in one exon in cases 16 and 17. Each PCR product (30 ng), including the regions harboring mutations, was ligated with the vector (50 ng) and transformed into cells using the Competent High JM109 Kit (Toyobo, Osaka, Japan). The DUOX2 sequences of the patients' relatives were analyzed after obtaining informed consent. Cis or trans arrangement of the multiple variations was determined by analyzing the DUOX2 genes of the patients' parents.

To analyze the genes encoding TPO, NIS and DUOXA2, all coding exons and surrounding intronic sequences were amplified by PCR and were sequenced directly as described previously $(4,6,9,13)$.

Eleven of the 25 patients $(8,10,11,13,14,16,17,20$, 21,23 , and 25), who were enrolled in the study after July 2012, were subjected to mutation screening for DUOX1, DUOX2, DUOXA1, DUOXA2, GNAS, FOXE1, IYD, NIS, NKX2-1, NKX2-5, PDS, PAX8, TG, TPO and TSHR using the
Haloplex Target Enrichment System (Agilent Technologies, Palo Alto, CA, USA). The library was sequenced as 150-bp paired-end reads on a MiSeq next-generation sequencer (Illumina, San Diego, CA, USA). Nucleotide alterations were called by the Surecall system (Agilent Technologies) and SAMtools 0.1.17 software (http:// samtools.sourceforge.net) and were confirmed by Sanger direct sequencing.

The functional role of seven DUOX2 variants (p.D115Y, p. A649E, p.V779M, p.E879K, p.R885Q， p.L1343F, and p.R1492H) and three polymorphisms (p.138L, p.H678R, and p.L1067S) were evaluated by using three different bioinformatics programs (PolyPhen-2 Polymorphism Phenotyping v2 (http://genetics.bwh. harvard.edu/pph2/), Mutation Tester (http://www.mutationtaster.org) and SIFT (http://sift.jcvi.org)), which predict the possible impact of an amino acid substitution on the structure and function of human proteins using straightforward physical and evolutionary comparative considerations.

\section{Results}

\section{Identification of variants by DUOX2 sequencing}

All patients had biallelic DUOX2 variants. Twenty-one distinct variants were detected (Table 1). The novel variants are shown in Supplementary Figures 1, 2, 3, 4, 5, 6 and 7, see section on supplementary data given at the end of this article. Variants p.V779M (rs145061993) and p.R1492H (rs147945181 and rs144543420) were previously reported in SNP databases. These novel seven variants have not been reported as polymorphisms in the Japanese population. The allelic frequencies of these variants in Japanese individuals are likely to be $<0.01$ (Human Genetic Variation database, http://www.genome. med.kyoto-u.ac.jp/SnpDB/). The most prevalent allele was c.2033A $>$ G (p.H678R); 10 of the 25 patients had this allele (Fig. 1).

None of the patients had biallelic mutations in any other genes involved in the generation of thyroid hormone (TPO, NIS, and DUOXA2). Furthermore, the 11 patients analyzed by next-generation sequencing only had biallelic mutations in DUOX2. Cases 10 and 11 (twins) also had a monoallelic mutation in TG (p.T1498M). Cases 16 and 17 (twins) had a heterozygous mutation in TG (p.R2585W). Both TG variants were reported on the web as rare and probably damaging variants by PolyPhen-2 (http://evidence.pgp-hms.org/TG-T1498M, http://evidence. pgp-hms.org/TG-R2585W). All the parents of the patients 


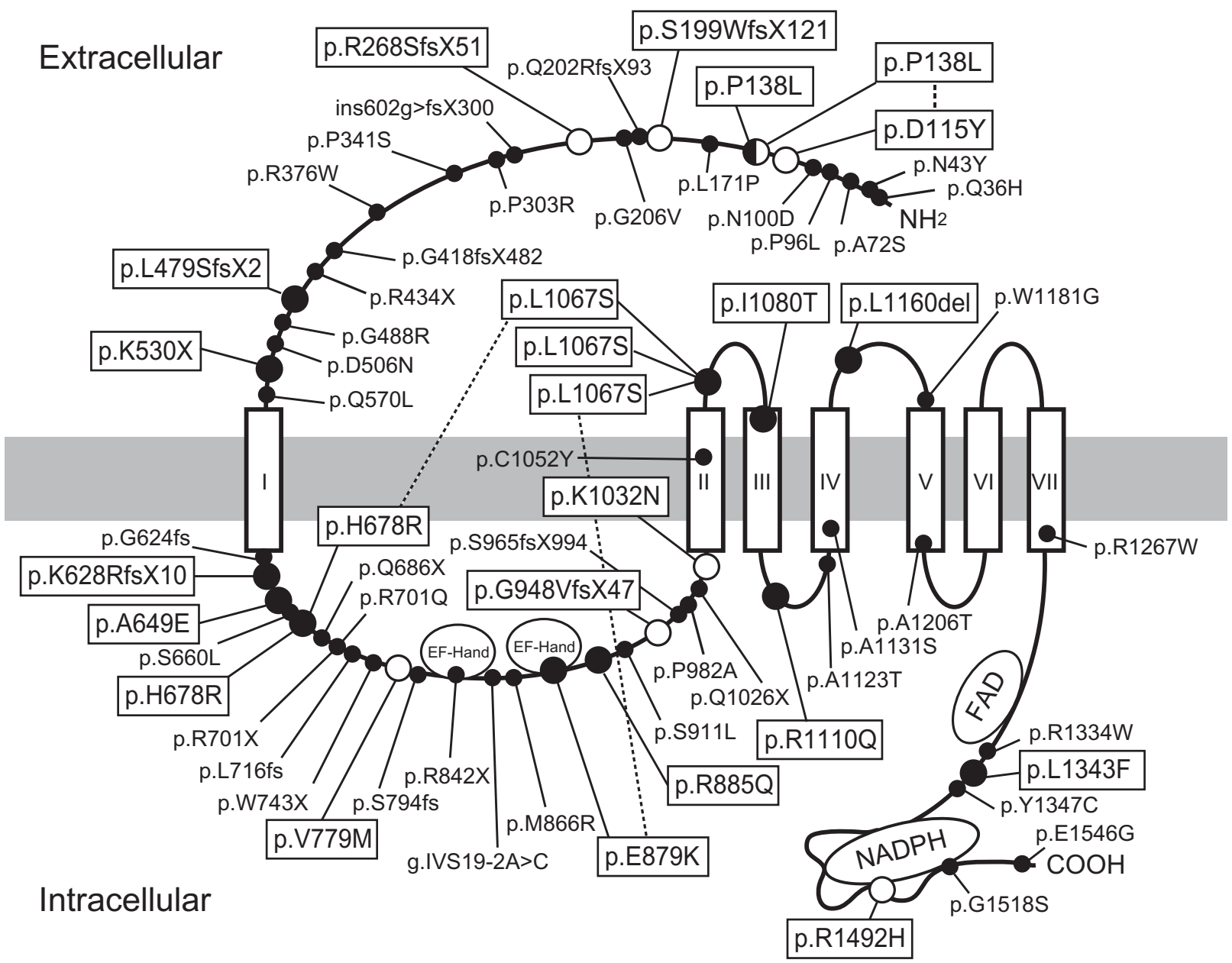

\section{Figure 1}

The 21 mutations detected in this study are shown on a topological model of the DUOX2 protein, which was based on structural data from De Deken et al., Vigone et al. and Maruo et al. $(10,13,19)$. The locations of all DUOX2 mutations reported to date $(8,13,14,15,16,19,20,21,22,23,24,25,26$, $27,28)$ are shown as small closed circles indicated by arrows with small characters. The locations of the 21 mutations detected in

included in this study are carriers of monoallelic DUOX2 mutations. They did not have a history of hypothyroidism.

\section{Prediction of the functional effects of missense variants in DUOX2 by in silico assays}

Prediction of the functional effects of the seven missense variants by in silico assays showed that p.D115Y, p. A649E, p.E879K, p.R885Q and p.R1492H were probably damaging and that p.V779Q, p.I1080T and p.L1343F are possibly this study are shown as open boxes with large characters. The seven novel mutations found in the present work are shown as large open circles. The dotted lines indicate the linkages between the mutations. The relative positions of the calcium-binding (EF-hand), flavin adenine dinucleotide (FAD)-binding and NADPH-binding motifs are also indicated $(10,17)$.

damaging. The results suggested that the seven missense mutations likely have a disease-causing role. In contrast, the three polymorphic variants (p.P138L, p.H678R and p.L1067S) were not predicted to be disease-causing variants in the in silico assays (Table 3 ).

\section{Clinical course of patients with biallelic mutations in DUOX2}

After beginning L-thyroxine treatment, serum $\mathrm{TSH}, \mathrm{fT}_{3}$ and $\mathrm{fT}_{4}$ levels in most patients improved within 2 months. 
Table 3 Prediction of functional effects of missense variants of DUOX2 by in silico assays.

\begin{tabular}{l} 
Variant \\
\hline p.D115Y \\
p.A649E \\
p.V779M \\
p.E879K \\
p.R885Q \\
p.I1080T \\
p.L1343F \\
p.R1492H \\
p.P138L \\
p.H678R \\
p.L1067S
\end{tabular}

\begin{tabular}{l} 
Polyphen2 $^{\mathbf{a}}$ \\
\hline Probably damaging \\
Probably damaging \\
Possibly damaging \\
Probably damaging \\
Probably damaging \\
Possibly damaging \\
Possibly damaging \\
Probably damaging \\
Benign \\
Benign \\
Benign
\end{tabular}

\begin{tabular}{ll}
\hline & \\
\hline 0.99 \\
0.94 \\
0.895 \\
1.00 \\
0.998 \\
0.834 \\
0.831 \\
1.00 \\
0.031 \\
0.000 \\
0.025 \\
\hline
\end{tabular}

\begin{tabular}{l}
\hline Mutation tester \\
\hline Disease causing \\
Disease causing \\
Disease causing \\
Disease causing \\
Disease causing \\
Disease causing \\
Disease causing \\
Disease causing \\
Polymorphism \\
Polymorphism \\
No data
\end{tabular}

\begin{tabular}{lll}
\hline SIFT Human Protein & & SIFT Score \\
\cline { 1 - 1 } Damaging & 0 \\
Tolerated & & 0.07 \\
Damaging & & 0.01 \\
Damaging & 0.04 \\
Tolerated & 0.06 \\
Damaging & 0 \\
Damaging & 0 \\
Damaging & 0 \\
Tolerated & 0.06 \\
Tolerated & 1 \\
Tolerated & 0.85 \\
\hline
\end{tabular}

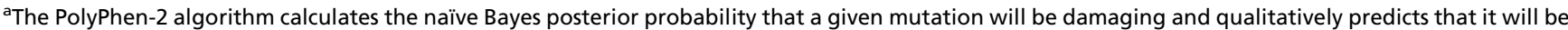
benign, possibly damaging or probably damaging, corresponding to posterior probability intervals $(0,0.2),(0.2,0.85)$, and $(0.85,1)$, respectively.

For most patients, the L-thyroxine dose could be reduced by about $2-4$ years of age, except for the twins in cases 10, 11 and 21 . Twenty-one of the 24 patients were able to receive reduced doses of L-thyroxine. Fourteen patients ranging in age from 2 years 1 month to 9 years 4 months were able to stop L-thyroxine treatment (median, 8 years 2 months). For the remaining seven patients, L-thyroxine was withdrawn by the conclusion of the study (ages 3-8 years). For the twins (cases 10 and 11) with compound heterozygous mutations p.R885Q and p.L1343F, it was necessary to increase the L-thyroxine dose to control the elevation of TSH and permanent $\mathrm{CH}$. For patient 21, with p.H678R and p.V779M, L-thyroxine also could not yet begin to be withdrawn. Elevated serum TG levels persisted after terminating L-thyroxine treatment. However, TG levels usually normalized after the patients reached 10 years of age (cases 1, 2, 3, 5, 12, 13, 15 and 20; Table 2). This indicates that the patients with DUOX2 mutations have subclinical hypothyroidism until puberty. After withdrawal of therapy, all patients were followed until recently (for 9 months-13 years) (Table 2). During puberty, none of the transient cases showed sign of hypothyroidism (elevation of TSH or reduction of $\mathrm{fT}_{4}$ level).

The oldest patient, who was compound heterozygous for p.L479SfsX2 and p.K628RfsX10, showed no signs of hypothyroidism after finishing treatment for the remainder of the study period (i.e., until she reached 18 years of age), and her TG level remained in the normal range.

The growth of the patients was within the normal range, and psychomotor development was also normal except for patient 5, who exhibited a mild learning disability (Table 2).

\section{Discussion}

After the discovery of the connection between DUOX2 deficiency and $\mathrm{CH}$, many patients with DUOX2 mutations have been reported (Fig. 1) $(8,13,14,15,16,19,20,21,22$, $23,24,25,26,27,28)$. However, some questions still remain. For example, which DUOX2 mutations result in permanent $\mathrm{CH}$ and which result in transient hypothyroidism? What is the natural course of DUOX2 deficiencies?

Moreno et al. (12) reported that biallelic DUOX2 mutations cause permanent $\mathrm{CH}$ and monoallelic mutations cause transient $\mathrm{CH}$. However, there is a manuscript supporting the hypothesis that carriers of monoallelic mutations only suffer from transient $\mathrm{CH}$ (29). This recent study also suggested the involvement of a monoallelic variant of DUOX2 in the development of transient $\mathrm{CH}$. Other reports have shown that biallelic DUOX2 mutations cause mild to moderate $\mathrm{CH}(13,14,15$, $16,19,22,30)$. Recently, some studies have shown that DUOX2 defects are the predominant cause of $\mathrm{CH}$ associated with dyshormonogenesis $(13,14,15,16,25,26)$.

In our analysis of 25 Japanese patients with $\mathrm{CH}$ carrying biallelic DUOX2 mutations, 13 patients finished L-thyroxine replacement therapy prior to reaching 10 years of age, and eight patients, ranging from 3 to 8 years old at the end of the study period, were receiving reduced L-thyroxine doses. This result indicates that in the Japanese population, DUOX2 mutations can cause transient $\mathrm{CH}$, even combinations of inactivation mutations (i.e., nonsense and frameshift mutations) as observed in family 1 (cases 1, 2, 3, and 4) and case 19. Moreover, case 4 in family 1 , with compound heterozygous p.L479SfsX2 and p.K628RfsX10 mutations, showed only transient hyperthyrotropinemia, whereas the patient's three elder 
sisters showed apparent transient $\mathrm{CH}$. These results indicate that DUOX2 mutations cause mild to moderate forms of hypothyroidism, including transient $\mathrm{CH}$ and transient hyperthyrotropinemia. A possible mechanism by which DUOX2 mutations cause transient $\mathrm{CH}$ is as follows. Two dual oxidases in thyrocytes, DUOX1 and DUOX2, generate $\mathrm{H}_{2} \mathrm{O}_{2}$ for organification and coupling of tyrosine $(10,17)$. However, the expression levels of these two DUOXs differ; the DUOX1 expression level is one-fifth that of DUOX2 (31). Even if DUOX2 enzyme activity is lost, a low level of $\mathrm{H}_{2} \mathrm{O}_{2}$ may be maintained by DUOX1 throughout life. The requirement for thyroid hormone during the neonatal period $(10-15 \mu \mathrm{g} / \mathrm{kg})$ is $5-7$ times higher than that of adults $(2 \mu \mathrm{g} / \mathrm{kg})(32,33)$; then, the thyroid hormone requirement gradually decreases after the infantile period. The $\mathrm{H}_{2} \mathrm{O}_{2}$ supply from DUOX1 alone may be inadequate during the neonatal and infantile periods, so individuals with DUOX2 deficiencies are liable to show signs of $\mathrm{CH}$. As the thyroid hormone requirement decreases over the course of development, DUOX1 production of $\mathrm{H}_{2} \mathrm{O}_{2}$ is sufficient to maintain thyroid hormone synthesis, irrespective of DUOX2 mutations (Fig. 2). However, the members of Family 1 were not analyzed by next-generation sequencing. The presence of other unidentified defects may be involved in the difference in the severity.

In addition to the frameshift mutations (p.S199 WfsX121, p.R268SfsX51 p.L479SfsX2, p.K628RfsX10, and p.G948VfsX47) and the one amino acid deletion variant (p.L1160del), prediction of the functional effect by in silico assays suggested that seven of the missense variants - p.D115Y, p. A649E, p.V779M, p.E879K, p.R885Q, p.L1343F, and p.R1492H - were disease-causing variants. In contrast, the in silico assays suggested that the three polymorphic variants - p.P138L, p.H678R, and p.L1067S - were silent polymorphisms. This was consistent with a previous expression study (25). Previous studies showed that p.H678R did not reduce $\mathrm{H}_{2} \mathrm{O}_{2}$ production $(14,34)$. The most prevalent variant detected in this study was p.H678R. Previously, Narumi et al. (14) reported that this variant is a polymorphism in the Japanese population and that it has an interactive effect with other mutations leading to a mild phenotype. Therefore, patients who are compound heterozygous for p.H678R and other pathological variants might exhibit mild clinical manifestations due to DUOX2 defects. However, even patients with a combination of nonsense and frameshift mutations (i.e., cases 1, 2, 3, 4 and 19) showed transient $\mathrm{CH}$. The location of the mutations within the DUOX2 protein varied (Fig. 1). Moreover,

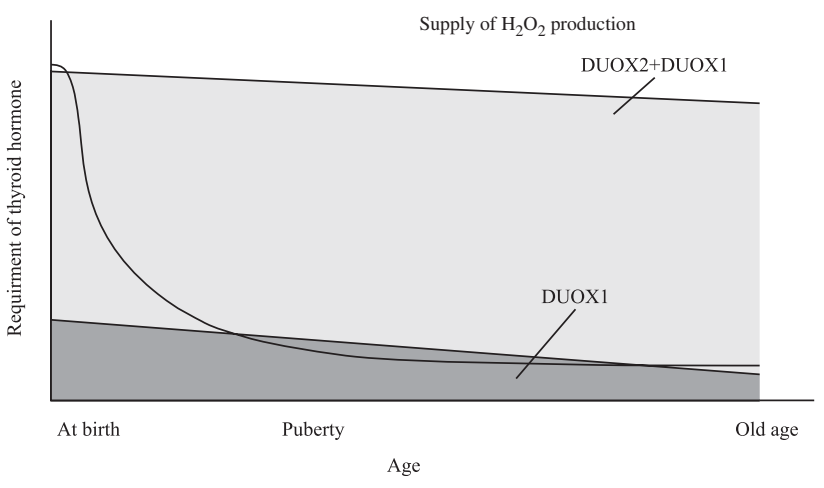

Figure 2

The proposed mechanism underlying the development of transient $\mathrm{CH}$ due to DUOX2 defects. Two dual oxidases, DUOX1 and DUOX2, generate $\mathrm{H}_{2} \mathrm{O}_{2}$ for thyroid hormone production in thyrocytes. The amount of DUOX1 expressed is one-fifth that of DUOX2. If DUOX2 enzyme activity is lost, a low level of $\mathrm{H}_{2} \mathrm{O}_{2}$ is maintained by DUOX1 throughout life. The requirement for thyroid hormone during the neonatal period is 5-7 times higher than it is in adults, and it gradually decreases after the infantile period. The $\mathrm{H}_{2} \mathrm{O}_{2}$ supply from DUOX1 alone may be inadequate during the neonatal and infantile periods, so that carriers with DUOX2 deficiency exhibit hypothyroidism. As the thyroid hormone requirement decreases, the production of $\mathrm{H}_{2} \mathrm{O}_{2}$ by DUOX1 is sufficient to maintain thyroid hormone synthesis, irrespective of the DUOX2 genotype.

some patients (cases 10 and 11; compound heterozygotes p.R885Q and p.L1343F alleles) also exhibited permanent $\mathrm{CH}$. Therefore, it is difficult to detect associations between DUOX2 mutations and prognosis.

The results of the next generation sequencing of 11 patients, showing no other biallelic mutations in thyroid hormonogenesis genes, indicated that a DUOX2 defect is an important cause of hypothyroidism. However, modulators other than the genetic polymorphism are thought to exist that lead to the variety observed in the patients' clinical manifestations. Permanent $\mathrm{CH}$ cases (cases 10 and 11) had biallelic mutations in DUOX2 and a monoallelic mutation in $T G$. However, cases 16 and 17 with transient $\mathrm{CH}$ had a combination of the biallelic mutations in DUOX2 and the monoallelic mutation in TG. A recent report revealed that the combination of biallelic mutation in DUOX2 with the monoallelic mutation in a different thyroid hormone-generating enzyme might cause $\mathrm{CH}$. (35). However in some cases, the combination of biallelic mutation in DUOX2 with the monoallelic mutation in a 
different thyroid hormone-generating enzyme might cause permanent $\mathrm{CH}$.

Another factor in patients with DUOX2 deficiencies that might affect the differential prognosis between transient $\mathrm{CH}$ in Japanese and permanent $\mathrm{CH}$ in Europeans is the iodine content in soil. In Europe, the iodine content in the soil is low, whereas in Japan, it is plentiful (36). This difference might explain the different phenotypes observed in Europe and Japan, as a higher iodine supply might increase thyroid hormone production.

In our study, there was an unbalanced sex ratio, with 21 females and four males. It was not clear why such sex-based differences occurred. However, in family 1 (compound heterozygous for p.L479SfsX2 and p.K628RfsX10), the three elder sisters showed apparent transient hypothyroidism, whereas the younger brother showed only transient hyperthyrotropinemia. These cases suggested that the symptoms of the DUOX2 defect might be different in each individual, even in those with same mutations.

TG levels were elevated for 3-10 years after the L-thyroxine treatments ended (Table 2). After the start of puberty, when the L-thyroxine requirement is reduced to that of adults, the TG level improved to within the normal range. The delayed improvement of the TG level suggests that patients with DUOX2 defects might exhibit insufficient thyroid hormone production. After stopping the L-thyroxine treatment, TG levels improved, and hypothyroidism did not recur in the patients from puberty to 18 years of age (cases $1,2,3,5$, and 20). However, Ohye et al. showed that a patient who was homozygous for p.R1110Q developed acquired goiterous hypothyroidism (23). The goiter appeared when the patient was in her $20 \mathrm{~s}$, and hypothyroidism developed when the patient was in her $40 \mathrm{~s}$. Abe et al. (22) reported a 39-year-old mother with a homozygous mutation (p.H678R) and a mild phenotype that also showed elevated TSH $(15.6 \mu \mathrm{U} / \mathrm{ml})$. Accordingly, our patients with transient $\mathrm{CH}$ might develop hypothyroidism later in life. Given the increased need for thyroid hormone during pregnancy, pregnant women with a history of transient $\mathrm{CH}$ due to bialellic mutations in DUOX2 should be observed carefully, especially after withdrawal of L-thyroxine. Further observations are necessary to clarify the clinical course of DUOX2 defects.

In this study, the growth and development of patients identified by neonatal screening were largely normal. In contrast, a previous report of Turkish patients with homozygous p.R434X showed mental retardation due to delayed diagnosis at 6 months of age (26). Therefore, early diagnosis by neonatal screening programs and early treatment are important for patients with DUOX2 defects, even for transient $\mathrm{CH}$.

\section{Conclusion}

Japanese patients with DUOX2 defects primarily showed transient $\mathrm{CH}$. Many patients completed L-thyroxine replacement therapy before reaching puberty. As some patients showed permanent $\mathrm{CH}$ and transient hyperthyrotropinemia, DUOX2 mutations might be associated with various phenotypes. To clarify the phenotypes and clinical course of DUOX2 mutations, additional data and patient observations are necessary.

\section{Supplementary data}

This is linked to the online version of the paper at http://dx.doi.org/10.1530/ EJE-15-0959.

\section{Declaration of interest}

The authors declare that there is no conflict of interest that could be perceived as prejudicing the impartiality of the research reported.

\section{Funding}

This work was partly supported by grants-in-aid for scientific research from the Ministry of Education, Science, and Culture of Japan (grant numbers 24659495, 15K09710).

\section{Acknowledgements}

We thank M Suzaki of the Central Research Laboratory, Shiga University of Medical Science, for technical assistance. The sequence data for seven novel DUOX2 alleles have been submitted to the DDBJ/EMBL/GenBank databases under the accession numbers $A B 779680$ (c. [343T $>G ; 413 C>T$ ], p.[D115Y;P138L]), AB779681 (c.596delC, p.S199WfsX121), AB779682 (c.804delG, p.R268SfsX52), AB779686 (c.2840delG, p.G948VfsX48), LC012880 (c.2335G >A, p.V779M), LC012881 (c.3096G >C, p.K1032N) and LC012883 (c.4475G >A, p.L1492H).

\section{References}

1 Medeiros-Neto G, Knobel M, DeGroot LJ. Genetic disorders of the thyroid hormone system. In: Baxter JD, eds. In Genetics in Endocrinology. pp. 375-402. Philadelphia: Lippincott Williams \& Wilkins, 2002.

2 Knobel M \& Medeiros-Neto G. An outline of inherited disorders of the thyroid hormone generating system. Thyroid 200313 771-802. (doi:10.1089/105072503768499671)

3 de Felice M \& di Lauro R. Thyroid development and its disorders: genetics and molecular mechanisms. Endocrine Reviews 200425 722-746. (doi:10.1210/er.2003-0028)

4 Abramowicz MJ, Targovnik HM, Varela V, Cochaux P, Krawiec L, Pisarev MA, Propato FV, Juvenal G, Chester HA \& Vassart G. Identification of a mutation in the coding sequence of the human 
thyroid peroxidase gene causing congenital goiter. Journal of Clinical Investigation 199290 1200-1204. (doi:10.1172/JCI115981)

5 Ieiri T, Cochaux P, Targovnik HM, Suzuki M, Shimoda SI, Perret J \& Vassart G. A 3-prime splice site mutation in the thyroglobulin gene responsible for congenital goiter with hypothyroidism. Journal of Clinical Investigation 199188 1901-1905. (doi:10.1172/ JCI115513)

6 Fujiwara H, Tatsumi K, Miki K, Harada T, Miyai K, Takai S \& Amino N. Congenital hypothyroidism caused by a mutation in the $\mathrm{Na}(+) / \mathrm{I}(-)$ symporter. Nature Genetics 199716 124-125. (doi:10.1038/ ng0697-124)

7 Scott DA, Wang R, Kreman TM, Andrews M, McDonald JM, Bishop JR, Smith RJH, Karniski LP \& Sheffield VC. Functional differences of the PDS gene product are associated with phenotypic variation in patients with Pendred syndrome and non-syndromic hearing loss (DFNB4). Human Molecular Genetics 20009 1709-1715. (doi:10.1093/hmg/ 9.11.1709)

8 Moreno JC, Bikker H, Kempers MJ, van Trotsenburg AS, Baas F, de Vijlder JJ, Vulsma T \& Ris-Stalpers C. Inactivating mutations in the gene for thyroid oxidase 2 (THOX2) and congenital hypothyroidism. New England Journal of Medicine 2002347 95-102. (doi:10.1056/ NEJMoa012752)

9 Zamproni I, Grasberger H, Cortinovis F, Vigone MC, Chiumello G, Mora S, Onigata K, Fugazzola L, Refetoff S, Persani L et al. Biallelic inactivation of the dual oxidase maturation factor 2 (DUOXA2) gene as a novel cause of congenital hypothyroidism. Journal of Clinical Endocrinology and Metabolism 200893 605-610. (doi:10.1210/ jc. 2007-2020)

10 De Deken X, Wang D, Many MC, Costagliola S, Libert F, Vassart G, Dumont JE \& Miot F. Cloning of two human thyroid cDNAs encoding new members of the NADPH oxidase family. Journal of Biological Chemistry 2000275 23227-23233. (doi:10.1074/jbc.M000916200)

11 Grasberger H \& Refetoff S. Identification of the maturation factor for dual oxidase. Evolution of an eukaryotic operon equivalent. Journal of Biological Chemistry 2006281 18269-18272. (doi:10.1074/jbc. C600095200)

12 Moreno JC, Klootwijk W, van Toor H, Pinto G, D'Alessandro M, Leger A, Goudie D, Polak M, Gruters A \& Visser TJ. Mutations in the iodotyrosine deiodinase gene and hypothyroidism. New England Journal of Medicine 2008358 1811-1818. (doi:10.1056/ NEJMoa0706819)

13 Maruo Y, Takahashi H, Soeda I, Nishikura N, Matsui K, Ota Y, Mimura Y, Mori A, Sato H \& Takeuchi Y. Transient congenital hypothyroidism caused by biallelic mutations of the dual oxidase 2 gene in Japanese patients detected by a neonatal screening program. Journal of Clinical Endocrinology and Metabolism 200893 4261-4267. (doi:10.1210/jc.2008-0856)

14 Narumi S, Muroya K, Asakura Y, Aachi M \& Hasegawa T. Molecular basis of thyroid dyshormonogenesis: genetic screening in population-based Japanese patients. Journal of Clinical Endocrinology and Metabolism 2011 96 E1838-E1842. (doi:10.1210/jc.2011-1573)

15 Rabbiosi S, Vigone MC, Cortinovis F, Zamproni I, Fugazzola L, Persani L, Corbetta C, Chiumello G \& Weber G. Congenital hypothyroidism with eutopic thyroid gland: analysis of clinical and biochemical features at diagnosis and after re-evaluation. Journal of Clinical Endocrinology and Metabolism 201398 1395-1402. (doi:10.1210/ jc.2012-3174)

16 Jin HY, Heo SH, Kim YM, Kim GH, Choi JH, Lee BH \& Yoo HW. High fequency of DUOX2 mutations in transient or permanent congenital hypothyroidism with eutopic thyroid glands. Hormone Research in Poediatrics 201482 252-260.

17 Dupuy C, Ohayon R, Valent A, Noel-Hudson MS, Deme D \& Virion Q. Purification of a novel flavoprotein involved in the thyroid NADPH oxidase. Journal of Biological Chemistry 1999274 37265-37269. (doi:10.1074/jbc.274.52.37265)
18 Corvilain B, van Sande J, Laurent E \& Dumont JE. The $\mathrm{H}_{2} \mathrm{O}_{2}$-generating system modulates protein iodination and the activity of the pentose phosphate pathway in dog thyroid. Endocrinology 1991128 779-785. (doi:10.1210/endo-128-2-779)

19 Vigone MC, Fugazzola L, Zamproni I, Passoni A, Di Candia S, Chiumello G, Persani L \& Weber G. Persistent mild hypothyroidism associated with novel sequence variants of the DUOX2 gene in two siblings. Human Mutation 200526 395. (doi:10.1002/ humu.9372)

20 Varela V, Rivolta CM, Esperante SA, Gruneiro-Papendieck L, Chiesa A \& Targovnik HM. Three mutations (p.Q36H, p.G418fsX482, and g.IVS19$2 \mathrm{~A}>\mathrm{C}$ ) in the dual oxidase 2 gene responsible for congenital goiter and iodide organification defect. Clinical Chemistry 200652 182-191. (doi:10.1373/clinchem.2005.058321)

21 Pfarr N, Korsch E, Kaspers S, Herbst A, Stach A, Zimmer C \& Pohlenz J. Congenital hypothyroidism caused by new mutations in the thyroid oxidase 2 (THOX2) gene. Clinical Endocrinology 200665 810-815. (doi:10.1111/j.1365-2265.2006.02672.x)

22 Ohye H, Fukata S, Hishinuma A, Kudo T, Nishihara E, Ito M, Kubota S, Amino N, Ieiri T, Kuma K et al. A Novel homozygous missense mutation of the dual oxidase 2 (DUOX2) gene in an adult patient with large goiter. Thyroid 200818 561-566. (doi:10.1089/thy.2007.0258)

23 Abe K, Narumi S, Suwanai AS, Hamajima T \& Hasegawa T. Pseudodominant inheritance in a family with non-autoimmune hypothyroidism due to biallelic DUOX2 Mutations. Clinical Endocrinology 201583 394-398. (doi:10.1111/cen.12622)

24 Wang F, Lu K, Yang Z, Zhang S, Lu W, Zhang L, Liu S \& Yan S. Genotypes and phenotypes of congenital goitre and hypothyroidism caused by mutations in dual oxidase 2 genes. Clinical Endocrinology 2014 81 452-457. (doi:10.1111/cen.12469)

25 Muzza M, Rabbiosi S, Vigone MC, Zamproni I, Cirello V, Maffini MA, Maruca K, Schoenmakers N, Beccaria L, Gallo F et al. The clinical and molecular characterization of patients with dyshormonogenic congenital hypothyroidism reveals specific diagnostic clues for DUOX2 defects. Journal of Clinical Endocrinology and Metabolism 2014 99 E544-E553.

26 Cangul H, Aycan Z, Kendall M, Bas VN, Saglam Y, Barrett TG \& Maher ER. A truncating DUOX2 mutation (R434X) causes severe congenital hypothyroidism. Journal of Pediatric Endocrinology \& Metabolism 201427 323-327.

27 Hoste C, Rigutto S, Van Vliet G, Miot F \& De Deken X. Compound heterozygosity for a novel hemizygous missense mutation and a partial deletion affecting the catalytic core of the $\mathrm{H}_{2} \mathrm{O}_{2}$-generating enzyme DUOX2 associated with transient congenital hypothyroidism. Human Mutation 201331 E1304-E1319. (doi:10.1002/ humu.21227)

28 Satoh M, Aso K, Ogikubo S, Yoshizawa-Ogasawara A \& Saji T. Hypothyroidism caused by the combination of two heterozygous mutations: one in the TSH receptor gene the other in the DUOX2 gene. Journal of Pediatric Endocrinology \& Metabolism 201528 657-661. (doi:10.1515/jpem-2014-0078)

29 Fu C, Zhang S, Su J, Luo S, Zheng H, Wang J, Qin H, Chen Y, Shen Y, $\mathrm{Hu} \mathrm{X}$ et al. Mutation screening of DUOX2 in Chinese patients with congenital hypothyroidism. Journal of Endocrinological Investigation 201538 1219-1224. (doi:10.1007/s40618-015-0382-8)

30 Yoshizawa-Ogasawara A, Ogikubo S, Satoh M, Narumi S \& Hasegawa T. Congenital hypothyroidism caused by a novel mutation of the dual oxidase 2 (DUOX2) gene. Journal of Pediatric Endocrinology \& Metabolism 201326 45-52. (doi:10.1515/jpem-2012-0082)

31 Moreno JC, Pauws E, van Kampen AH, Jedlickova M, de Vijlder JJ \& Ris-Stalpers C. Cloning of tissue-specific genes using serial analysis of gene expression and a novel computational subtraction approach. Genomics 200175 70-76. (doi:10.1006/geno.2001.6586)

32 Revised guidelines for neonatal screening programmes for primary congenital hypothyroidism. Working group on neonatal screening of 
the European society for paediatric endocrinology. Hormone Research 199952 49-52. (doi:10.1159/000023433)

33 LaFranchi S. Congenital hypothyroidism: etiologies, diagnosis, and management. Thyroid 19999 735-740. (doi:10.1089/thy. 1999.9.735)

34 De Marco G, Agretti P, Montanelli L, Di Cosmo C, Bagattini B, De Servi M, Ferrarini E, Dimida A, Freitas Ferreira AC, Molinaro A et al. Identification and functional analysis of novel dual oxidase 2 (DUOX2) mutations in children with congenital or subclinical hypothyroidism.
Journal of Clinical Endocrinology and Metabolism 201196 E1335-E1339. (doi:10.1210/jc.2010-2467)

35 Yoshizawa-Ogasawara A, Abe K, Ogikubo S, Narumi S, Hasegawa T \& Satoh M. Transient congenital hypothyroidism caused by compound heterozygous mutations affecting the NADPH-oxidase domain of DUOX2. Journal of Pediatric Endocrinology \& Metabolism. In press. (doi:10.1515/jpem-2014-0479)

36 Zimmermann MB, Jooste PL \& Pandav CS. Iodine-deficiency disorders. Lancet 2008372 1251-1262. (doi:10.1016/S0140-6736(08)61005-3)

Received 17 December 2015

Revised version received 7 January 2016

Accepted 7 January 2016 